\title{
Cytogenetic Analysis of Osmanabadi and Boer Breeding Bucks Breeds
}

\author{
S.C. Kokani*, V.D. Pawar, M.P. Sawane, M.M. Chopade and S.B. Khade \\ Department of Animal Genetics and Breeding, Bombay Veterinary College, Parel \\ Mumbai - 400012, India \\ *Corresponding author
}

\begin{abstract}
A B S T R A C T
Keywords

Karyotype, QFQ-

banding technique,

Osmanabadi buck,

Boer buck

Article Info

Accepted:

04 May 2018

Available Online:

10 June 2018

The cytogenetic study was conducted on total 30 breeding bucks of Osmanabadi and Boer breed (15 of each) to find out chromosomal abnormalities and to study QFQ- banding pattern of the chromosomes. Short term lymphocyte culture technique was used for the display chromosomal metaphases. The few slides were conventionally stained with 4 per cent giemsa stain and some were subjected for QFQ banding. The modal diploid chromosome number was $2 \mathrm{n}=60$ for breeding bucks of Osmanabadi and Boer breed of goat. There were 29 pairs of autosomes and one pair of sex chromosome in complement. The $\mathrm{X}$ chromosome was the largest acrocentric chromosome whereas the $\mathrm{Y}$ chromosome was the smallest dot like in majority of the metaphase examined however in few metaphases it was found to be sub metacentric in morphology. The QFQ banding pattern was obtained by using 0.5 per cent Quinacrine dihydrochloride solution for 20 minutes and karyotype was prepared as per ISCNDB, 2000. However, it was noted that the goat chromosome do not present the brilliant fluorescence. No marked chromosomal abnormalities were observed in the present study.
\end{abstract}

\section{Introduction}

Goat is an important livestock species of livestock for India. Around 80 per cent of the global goat population is found in developing countries. Amongst them, India ranks second in goat population with 125.7 million goats. At present there are 26 documented breeds of goat in India out of which4 breeds are found in Maharashtra. Osmanabadi is the important goat breed of Maharashtra found mainly in Osmanabad and Latur district. It is famous for quality of chevon and also known for better prolificacy. The dressing percentage varies from 45-50 per cent. Boer is a breed of goat that was developed in South Africa in the early 1900s for meat production. The Boer goat has a fast growth rate and making it one of the most popular breeds of meat goat in the world. Boer goats have a high resistance to disease and adapt well to hot and dry semi deserts. The kid of a proven fast-growing sire might weigh $36 \mathrm{~kg}$ at 90 days, while the kid of a poor-quality sire might weigh only $15 \mathrm{~kg}$ at 90 days.

Animal cytogenetics has several applications in animal improvement and chromosomal abnormalities associated with the reduced fertility, embryonic loss, foetal wastage and 
internal and external genital malformations. Many chromosomal abnormalities are known for lowered reproductive efficiency in goat and other livestock species due to the production of unbalanced gametes during meiosis. These abnormalities associated with reproductive failure have created concern in various countries and many of them have started regular chromosomal screening of breeder males to prevent substantial losses in animal production. The detection of chromosomal abnormalities in these domestic animals will prevent the rapid spread of chromosomal defects in subsequent generations.

In India breedable goats and sheep carrier for major structural cytogenetic defects are not been identified by Karyotyping and allowed for breeding through NS, AI or ET. So the chances of spreading the defects such as Reciprocal or Robertsonian translocation in the population and causing reduced fertility, early embryonic death, abortions, decreased litter size etc., has been increased. Identification of chromosomal defects is possible by using differential staining techniques such as G-, C-, R-, and Q- banding. Considering above facts, it becomes necessary to screen the breeding bucks for finding chromosomal abnormalities and ultimately preventing possible economic losses to the farmers.

\section{Materials and Methods}

The total 30 blood samples (15 of each breed) were collected from Osmanabadi and Boer bucks maintained at NARI, Phaltan DistSatara for the present study. The present research work was carried out at Genetic Investigation Laboratory (GIL), Department of Animal Genetics and Breeding, Bombay Veterinary College, Parel, Mumbai. Proper aseptic precautions were taken by cleaning the skin of the animal with 70 per cent ethanol, prior to the blood collection. About $5 \mathrm{ml}$ whole blood was drawn aseptically by puncturing external jugular vein into a vacutainer tube with net contents 150-200 I.U. of sodium heparin (Betcon Dickinson vacutainer systems).

The short term lymphocyte culture technique described by Moorhead et al., (1960) with some modifications was adopted for cytogenetic studies of bucks. In each $15 \mathrm{ml}$ culture tube $5 \mathrm{ml}$ RPMI- 1640 medium (GIBCO), Catalogue no. G11875-O85) was transferred then added about $1 \mathrm{ml}$ (10 to 20 per cent of culture) of fetal bovine serum (HYCLONE) catalogue no. SH3084.03) to provide essential nutrients, which may not be present in the synthetic medium. To stimulate mitosis phytohemagluttin (GIBCO) catalogue No. G132293) was added in each culture tube along with $40 \mu \mathrm{l}$ of antibiotic i.e. Penicillin (100 IU/ml of medium) and Streptomycin $(100 \mathrm{mg} / \mathrm{ml}$ of medium) combination (HIMEDIA) Catalogue no.A-002A) solution to avoid microbial contamination. Finally, about $0.5 \mathrm{ml}$ blood was added to each culture tube, respectively. The culture was then incubated at $37^{\circ} \mathrm{C}$ for a period of $72 \mathrm{hrs}$. After 70 hours of incubation, $75 \mu \mathrm{l}$ of spindle inhibitor colchicine (2 mg / $10 \mathrm{ml}$ ) (SIGMA) Catalogue No. C9754) was added to each culture tube; mixed gently and further incubated for 2 hours at $37^{\circ} \mathrm{C}$. After 72 hours tubes were centrifuged at $1000 \mathrm{rpm}$ for about 10 minutes. The supernatant was discarded leaving about $0.5 \mathrm{ml}$ cell button. $8 \mathrm{ml}$ of prewarmed hypotonic solution (0.075 M KCL) (SISCO) was added to these tubes and tubes were kept at $37^{\circ} \mathrm{C}$ in water bath for 20 minutes.

The tubes were removed from the water bath and freshly prepared chilled $1 \mathrm{ml}$ of fixative solution (3:1 methanol and glacial acetic acid) was added to each tube. These tubes were centrifuged at $1000 \mathrm{rpm}$ for 10 minutes and 
the supernatant hypotonic solution was removed to leave small whitish button of cells along with $0.5-1 \mathrm{ml}$ of hypotonic solution. Subsequently, 2-3 fresh fixative washes were given till the appearance of a whitish cell button at the bottom of the tube. The cell button was re-suspended in $0.5 \mathrm{ml}$ of fresh fixative for slide preparation and slides were air dried and labelled.

Few slides were stained with 4 per cent giemsa (HIMEDIA) solution for 45 minutes and washed and dried. The stained slides were mounted in DPX mountant. The remaining slides were kept for 20 minutes in 0.25 per cent quinacrine dihydrochloride (SIGMA) (Catalogue No. Q 2876) solution and rinsed with distilled water and kept for 30 seconds in another container of Mcllvaine buffer (Sodium dibasic phosphate+ citric acid) of $\mathrm{pH}$ 5.5-5.6.

The slides were mounted in the same mounting Mcllvaine buffer and observed under UV illumination with fluorescence microscope Olympus BX61.

\section{Results and Discussion}

Total 25 metaphases per sample were captured and analysed, the modal diploid chromosome number was found to be $2 n=60$. Twenty-nine pairs of acrocentric autosomes were observed and classified based on gradually decreasing length. A pair of sex chromosome comprised of the $\mathrm{X}$ chromosome this was the largest chromosome in the complement and the $\mathrm{Y}$ chromosome which was the smallest dot like in majority of the metaphase examined. However, in few metaphases it was found to be sub metacentric in morphology.

The banding of the chromosome of bucks were carried out using QFQ-banding technique. The QFQ banding protocol with few modifications described by Casperson et al., (1971) was adopted for banding of goat chromosomes. Different concentrations of
Quinacrine dihydrochloride and exposure time were tried and best bands were obtained at 0.25 per cent of Quinacrine dihydrochloride treatment for 20 minutes. In the present study Q- band pattern of both the breeds shows the same morphology and chromosome number which is $2 n=60,29$ pairs of autosomes and a pair of sex chromosome containing $X$ as a largest acrocentric and $\mathrm{Y}$ as the smallest sub metacentric chromosome in complement. The chromosomes are arranged according to Hansen (1973) and ISCNDB (2000) which is also applicable for cattle.

Presented findings of karyotyping were in accordance with Bhatia and Shankar (1991) who reported 29 pairs of acrocentric autosomes and 1 pair of sex chromosome in Gaddi breed of goat. Similar findings were reported by Saravanan et al., (2006) in Kanniadu goat and by Kasabe et al., (2009) in Berari goats. Sakaram et al., (2003) studied the karyotype of nondescript goat of Rohilakhand and found that $\mathrm{X}$ chromosome was the largest one, as like in the present study. Uma Devi et al., (2011) and Kumari et al., (2015) observed that the Y chromosome appears to be smallest and dot like in Mahabubnagar local goat breed and for Black Bengal goat breeds, respectively.

The previously published Q-, banding pattern of goat chromosomes (Hansen, 1973) was helpful for the Q- Banding research work attempted to match the obtained $\mathrm{Q}$ banding patterns of the goat. The $\mathrm{Q}$ banding pattern observed was more or less similar to that of published by Hansen in 1973. In present study the characteristics Q banded pattern observed, the first pair of chromosome showed intense band on proximal end, pale band on central region and medium intense band on distal end of the chromosome. Second pair of autosome was showed intense bands separated by pale bands pale telomere were observed (Fig. 1 and 2). 
Fig.1 QFQ banded karyotype of Osmanabadi buck

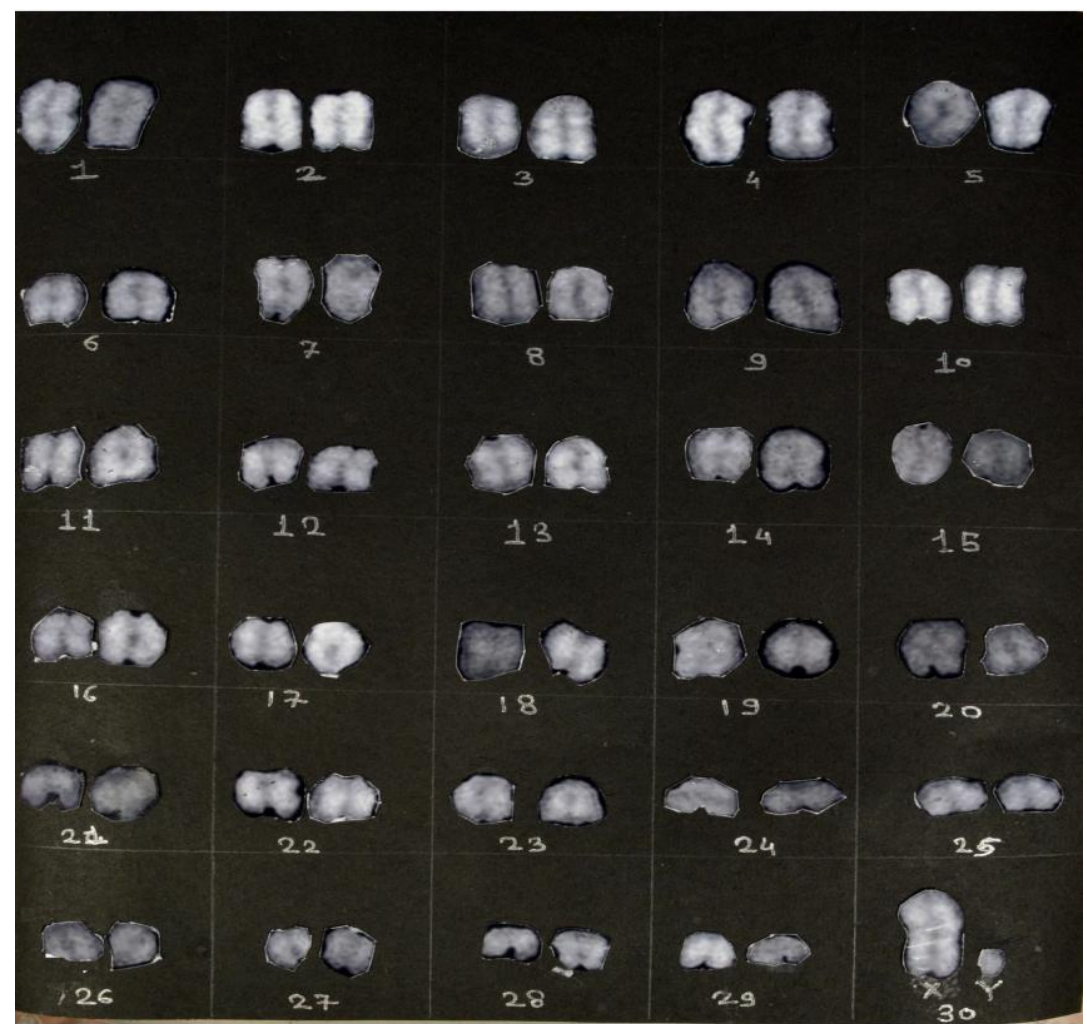

Fig.2 QFQ banded karyotype of Boer buck

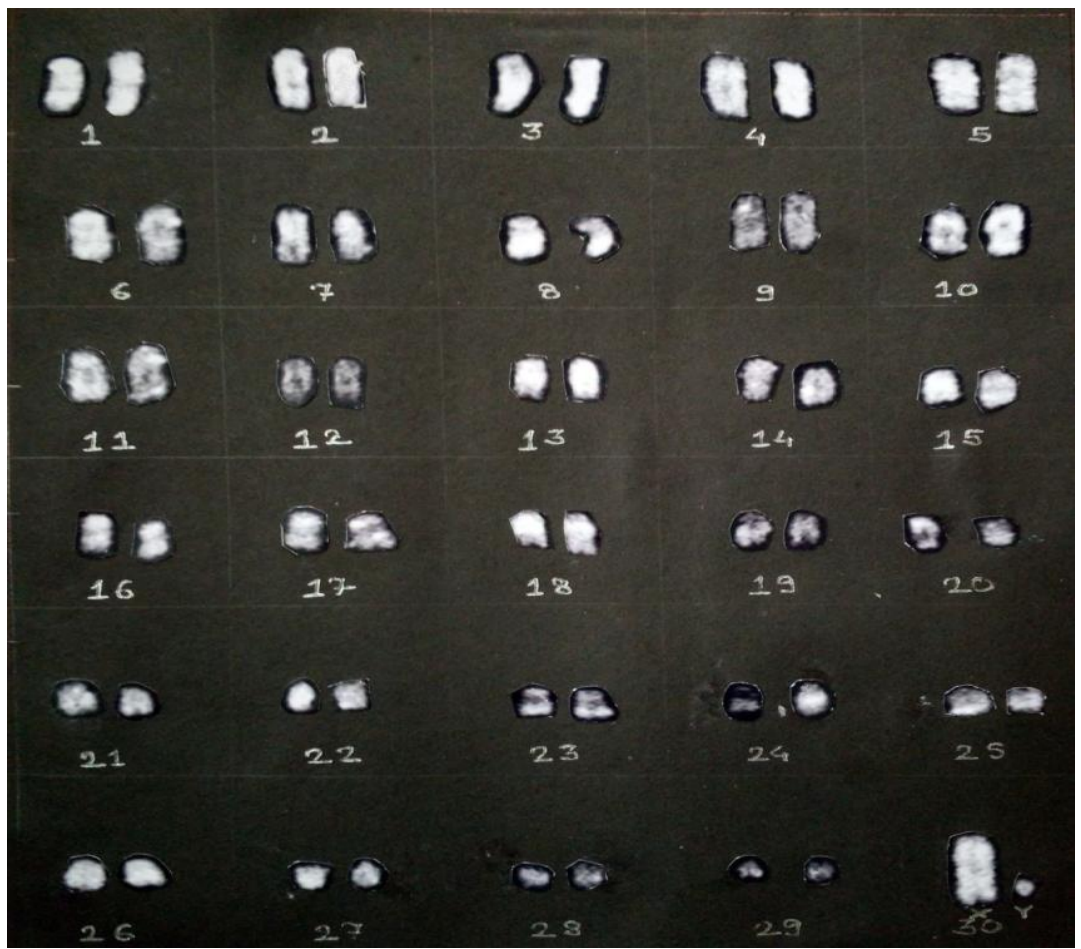


Chromosome No.3 showed narrow medium band on proximal region of chromosome, central intense and distal medium intensity band. Chromosome No. 4 showed medium intensity $Q$ positive bands whereas chromosome 5 presented negative medium band in central medium and pale telomere. The X chromosome was longest one with more intense band, presenting proximally intense band and centrally medium and distal end pale. The $\mathrm{Y}$ chromosome showed less intense fluorescence and the pale band on proximal region was observed. The QFQ banding technique was found to be simplest banding technique for the karyological evaluation of breeding bucks.

However, as per the published literature by Hansen (1973) the goat chromosomes do not present the brilliant fluorescence that makes the identification of the goat chromosomes a difficult task. We have experienced the similar situation in the present research work.

\section{Acknowledgment}

The authors are thankful to the NARI Phaltan for providing blood samples and essential data for study and Associate Dean, Bombay Veterinary College, Parel, Mumbai, Maharashtra, India, for providing essential facilities to carry out this investigation. Add-

\section{References}

Bhagat, S.B., Khade, A.S., Khade, S.B., Pawar, V.D., Umrikar, U.D., Sawane, M.P., Doiphode A.Y. (2014). J. Bombay Vet. College, Vol. 2 (1): 50-53.

Bhatia, S. and Shanker, V. (1991) Indian J. anim. Sci., 61(6):646-648.

Casperssson, T., G. Lomakka and L. Zech. (1971). Heriditas. 67: 89-102.

DiBerardino, G.P. DiMeo; D.S. Gallagher; H. Hayes and L. Iannuzi (2001). Cytogenetic cell genet, 92: 283-299.

Hansen, K.M. (1973) Hereditas, 73: 65-70.

ISCNDB (2000). International System for Chromosome Nomenclature of Domestic Bovid.

Kasabe, S. S., S. Z. Ali, M. P. Sawane and S. V. Kuralkar. (2009). Indian. J. small ruminants. 15(2): 257-261.

Kumari, N., B. Mandal, T. Oraon and S.K. Thakur. (2015). Indian journal of science and technology. 8 (28).

Sakaram, D., S.M. Deb, A. K. Sharma and R.L. Arora. (2003). Indain Vet. Med. J., 27: 29-31.

Saravanan, karthickeyan and A.D. Deshpande. (2006). Indian. J. Small ruminants. Pp. 16-20

Uma Devi, P., B. Ramesh Gupta, K. S. Devi, K. D. Lakshmi. (2011). Tamilnadu J. Vet. \& Ani. Sci. 7(6): 268-276.

\section{How to cite this article:}

Kokani, S.C., V.D. Pawar, M.P. Sawane, M.M. Chopade and Khade, S.B. 2018. Cytogenetic Analysis of Osmanabadi and Boer Breeding Bucks Breeds. Int.J.Curr.Microbiol.App.Sci. 7(06): 543-547. doi: https://doi.org/10.20546/ijcmas.2018.706.062 